\title{
A LETTER FROM THE EDITOR
}

Welcome to the inaugural issue of the Journal of Biomedical Optics. The JBO has been founded to become the world's premier journal archiving the applications of modern optics technology to biomedical research. During the past decade we have witnessed an explosion of optical and optoelectronic technologies. Measurements that are routine today were state-of-the-art or even impossible just a few years ago. New concepts and capabilities are rapidly migrating to biomedical applications, such as clinical sensing, optical tomography, and medical diagnostics.

The Journal of Biomedical Optics has its origin in the Biomedical Optics symposia organized by SPIE and the European Optics Society. Since 1985 the size of this conference has increased exponentially, from 19 papers in 1985 to 1138 papers in the Biomedical Optics and BiOS Europe symposia in 1995. This favorable result suggested the need for a peer-reviewed journal in this new area of interdisciplinary research.

The response to the first call for submissions to the Journal of Biomedical Optics has been remarkable. In just a short period of time there have been over 65 submissions from 21 countries. The submitted papers have been on a diverse range of topics: from basic optical spectroscopy to the more speculative topic of medical optical imaging. The first issue includes papers on the use of lasers in surgery, fluorescence sensing, multiphoton imaging, and near-field microscopy. These papers illustrate the breadth of topics covered by the JBO, which is the intent. The scope of the JBO includes all such applications of modern optics technology to medical research, diagnosis, and treatment.

As illustrated by the first issue of the JBO, it accepts regular peer-reviewed submissions, communications, and reviews. Future issues are expected to contain editorials or letters that discuss issues of current interest, and articles on the ethical and legal issues related to the introduction of new optical technologies to health care.
Plans are already in place for future reviews and special issues. Special issues are being prepared by Dr. Barbara Pierscionek on "Light Scatter and Fluorescence of the Eye Lens," Dr. Rebecca Richards-Kortum on "In Situ Analysis of BiologicalSamples," and Drs. Marco Ferrari, David Benaron, and David Delpy on "Clinical Near Infrared Spectroscopy/Imaging." Dr. Winifred Denk has agreed to write a review on "Multiphoton Fluorescence Microscopy," and Dr. Leon Goldman a review on the "History of Lasers in Medicine." All the JBO editors will be pleased to receive your suggestions for reviews or special issues on your specialized area of research. Reviews and special issues are intended to provide the JBO readers with a detailed summary of a topic, which is preferable to an encyclopedic summary.

The JBO is somewhat unique as a scientific journal in that the covered topics are not only of academic interest, but also have immediate commercial applications. For this reason, the JBO intends to have scientists from private industry on its editorial board, of course with due consideration of proprietary information. I thank those corporations that have already become corporate sponsors, and I invite all companies and institutions involved in biomedical optics to become sponsors of the JBO.

I wish to offer my special thank you to the many individuals who have contributed to the successful start-up of the JBO. I thank all members of the Editorial Board for their efforts in soliciting manuscripts and seeing them through the review process. I thank Mr. Eric Pepper and Mr. Mohan Nair in the SPIE office for their exceptional skills in organizing the JBO from ground zero, and Ms. Mary Rosenfeld in this office, whose combined efforts made the first issue possible.

Joseph R. Lakowicz Center for Fluorescence Spectroscopy December 1995 